\title{
New approaches to old age
}

\section{To truly understand ageing, we must look beyond the diseases of old age.}

\section{Leonard Hayflick}

the he study of biological ageing was once the provenance of charlatans and snake-oil merchants. Over the last third of the twentieth century, however, it moved into the mainstream of biology. This change was probably driven more by the politics of the explosion in the numbers of old people in developed countries than by any advance in our understanding of the biology of ageing.

The purpose of much biomedical research is to increase life expectancy, and the twentieth century saw huge successes in this venture. In the past hundred years, life expectancy at birth in developed countries has increased from about 48 to 76 - the same gain that occurred over the previous 1,900 years. The average life expectancy at birth in ancient Rome of 20 resulted from the enormous number of deaths in early childhood, not unlike the present demographics in developing countries. It is the control of infectious diseases of the young that explains the increase in life expectancy during the twentieth century. Older people have benefited less because the chronic diseases to which they are more predisposed, such as cancer and cardiovascular disease, have been less yielding to biomedical research.

But this progress has neither advanced nor resulted from our understanding of ageing. Everyone knows the difference between the diseases that occur in early life and childhood development itself. Yet failure to distinguish between the diseases of old age and the ageing process is widespread even in the scientific community. The virtual resolution of poliomyelitis, acute lymphocytic leukaemia, Wilms' tumours and irondeficiency anaemia did not increase our knowledge of childhood development; similarly, the resolution of the leading causes of death in old age - cardiovascular disease, stroke and cancer - are unlikely to advance our knowledge of the ageing process.

Changes attributable to disease can be distinguished from age changes because, unlike any disease, ageing occurs in virtually every species and only after reproductive success. Animals removed from the wild and protected by humans will undergo changes with age even though their species may have not experienced ageing for thousands or millions of years.

Humans, and the pet and zoo animals that we choose to protect, are the only species in which large numbers experience ageing. In the absence of human impacts on their ecological niches, ageing in numbers proportional to those seen in humans simply does not occur in wild animals. The expression of age changes is not essential for the survival of any species. Humans have survived, and sometimes flourished, with a life expectancy at birth of 20 or 30 for more than 99.9 per cent of our time on the planet. Prehistoric human remains have rarely, if ever, revealed ages greater than about 50 .

Human ageing has revealed itself because modern humans have escaped many causes of death not only in early life but long after reproducing. In so doing, we have unmasked a process for which evolution never prepared us. One might conclude that ageing is an artefact of civilization.

One example of the consequences for science policy of the failure to distinguish

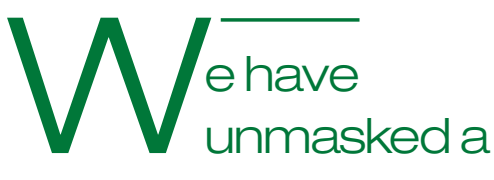

process for which

evolution never prepared us. One might conclude that ageing is an artefact of civilization. research on age-associated diseases from research on the fundamental biology of ageing is that it is virtually impossible to raise funds for research on ageing, because in the minds of policy-makers and the public no one suffers or dies from it. The general belief is that we suffer and die from the diseases that occur during the ageing process. Yet these diseases occur because age changes increase our vulnerability to what is ultimately written on death certificates.

More than half of the budget of the US National Institute on Aging is spent on Alzheimer's disease. Yet its elimination will have only a trivial impact on life expectancy and will not advance our knowledge of the fundamental biology of ageing. Greater attention must be given to a question that is rarely posed, yet which is capable not only of advancing our fundamental knowledge of ageing but also our understanding of ageassociated diseases: why are old cells more vulnerable to disease than young cells?

The resolution of all causes of death currently written on the death certificates of those older than 65 will result in an increase in life expectancy of only about 15 years. An increase in our knowledge of how age changes occur does not put a 15 -year limit on what is possible.

Leonard Hayflick is in the Department of Anatomy, University of California, San Francisco, PO Box 89, The Sea Ranch, California 95497, USA.

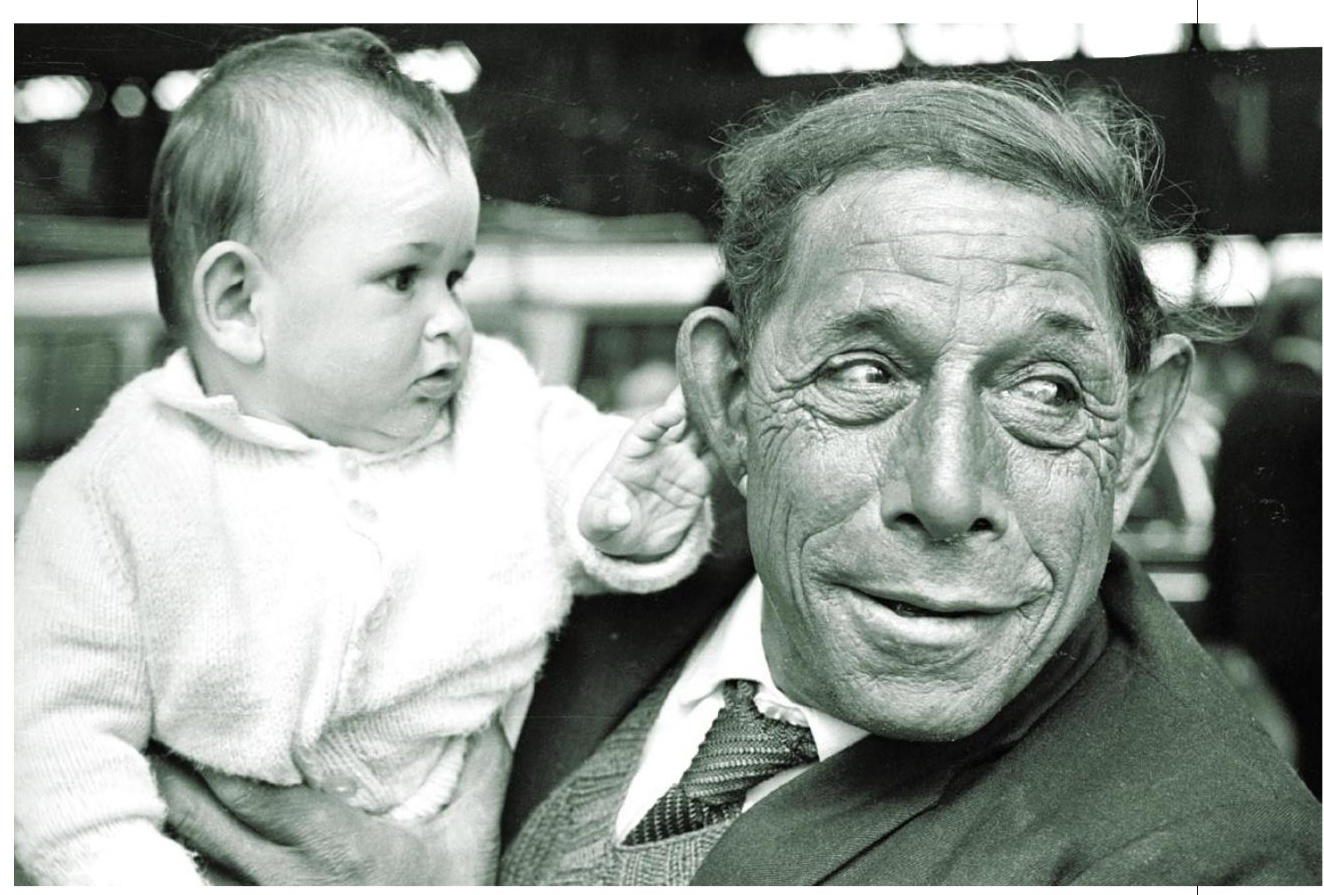

Generation gap: no one confuses child development and disease, but this is not true for the end of life. 